\title{
Prognostic factors and therapeutic perspectives of meningeal carcinomatosis in patients with breast cancer: A case report and review of the literature
}

\author{
Francesca Marasciulo*, Marialuisa Meacci, Guglielmo Fumi, Luca Marcomigni, Roberta Porreca and Fausto Roila \\ Medical Oncology Department, Azienda Ospedaliera - Universitaria Santa Maria della Misericordia, Perugia, Italy
}

\begin{abstract}
The incidence of meningeal carcinomatosis is progressively increasing as a consequence of the greater clinical control of secondary neoplasms from which it develops;

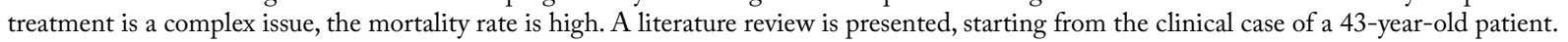

\section{Introduction}

Meningeal carcinomatosis is an increasingly frequent complication that develops late in patients with advanced, solid (mammary, pulmonary, melanoma) or hematological (lymphoma, leukemia) neoplasms [1]. It is often related to breast cancer (lobular carcinoma, triple negative subtype and HER-2 positive) [2] and to the adenocarcinoma histotype. Clinical manifestations are secondary to an obstruction to the flow of cerebrospinal fluid and/or to a direct infiltration by tumor cells of the leptomeninges (arachnoid and pia mater) with dissemination to the subarachnoid space [3]. In most cases symptoms are the consequence of increased intracranial pressure although they may also result from obstruction of cerebral vessels, altered cerebrospinal metabolism or direct infiltration of the brain parenchyma [1]. Diagnosis is performed by rachicentesis and/ or radiological examinations; MRI has shown superiority over other techniques. As treatment has not yet been standardized, it would be advisable to individualize it in each patient with regard to their underlying pathology, comorbidities and ability to respond to therapy [2]. In particular, the options in breast cancer include radiotherapy, intrathecal and systemic chemotherapy [4]. Prognosis is generally poor with a mean total survival of about 3-4 months from diagnosis, with better results in hematological and treatment-sensitive solid cancers [1].

\section{Clinical case}

The 43-year-old patient came to our attention due to a marked weakness in the lower limbs with inability to maintain an upright posture, headache and difficulty in urination. Remote medical history included: triple negative multicenter poorly differentiated ductal left breast cancer treated with left nipple-sparing mastectomy with ipsilateral axillary lymph node dissection and prosthetic reconstruction (January 2011), adjuvant chemotherapy according to the AC scheme for 4 cycles (until May 2011) followed by classic CMF for 3 cycles (until August 2011); Luminal B infiltrating ductal right breast cancer treated with neoadjuvant chemotherapy (until January 2019), nipple sparing mastectomy with sentinel lymph node biopsy, ipsilateral axillary dissection with breast reconstruction, insertion of right submuscular prosthesis and replacement of left breast prosthesis, followed by radiotherapy on the regional wall and lymph nodes and precautionary hormone therapy with LHRH analogue with exemestane (June 2019); prophylactic videolaparoscopic bilateral adnexectomy, suspending analogue LHRH and continuing exemestane (January 2020); brain metastases treated with panencephalic radiotherapy (April 2020). Following the onset of headache, nuchal rigidity, deficiency of the VII and V cranial nerve with central semeiology and weakness of the lower limbs, she was admitted in May 2020 to the Medical Oncology department of our hospital. The neurological physical examination showed the presence of weakness of the lower limbs with inability to maintain the upright and sitting position with positivity to the Mingazzini test, accompanied by paresthesia and a painful sensation that radiated from the occipital site to the lower limbs and, during hospitalization, also to the left upper limb. There was also a modest deviation of the labial rim to the left with asymmetry of the eyelid openings (right>left) and dysesthesia on the right half face. The remining clinical objectivity was normal. It was therefore decided to increase the dose of corticosteroids to $8 \mathrm{mg}$ TID and painkilling therapy (morphine and pregabalin) was set with progressive benefit. During hospitalization a series of tests were performed including an MRI of the brain and raquis with contrast medium. This showed the presence of pathological contrast impregnation of the internal acoustic canals and of the $\mathrm{V}$ cranial nerve, both in the cisternal site and in Merkel's cavity bilaterally, in correspondence of the medullary cord. In particular, this was evident in the cauda equina, which confirmed the diagnosis of leptomeningeal carcinomatosis. Carboplatin and paclitaxel chemotherapy was started (May 2020) without complications. The

${ }^{\star}$ Correspondence to: Francesca Marasciulo, Medical Oncology Department, Azienda Ospedaliera - Universitaria Santa Maria della Misericordia, S. Andrea delle Fratte, 06156 Perugia (PG), Italy, Tel: 3661765864; E-mail: fmara@myuax.com

Key words: chemotherapy, meningeal carcinomatosis, metastatic breast cancer, performance status, radiotherapy

Received: December 16, 2020; Accepted: December 23, 2020; Published: December 28, 2020 
patient was transferred to a hospice with progressive worsening of her clinical condition, until her death a few days later.

\section{Discussion and review of the literature}

The treatment of carcinomatous meningitis is complex, given the patient's poor ability to respond to therapy. In most cases the goal is to stabilize and/or improve the quality of life and extend survival [3]. A standard treatment scheme has not yet been formulated, but among the existing options are radiotherapy, intrathecal chemotherapy and systemic chemotherapy [4], which can be used according to individual needs. Although the prognosis varies according to type, degree of neoplasm and characteristics of the individual, some parameters can help differentiate high-risk patients (low performance status, presence of neurological deficits, advanced disease) from low-risk ones (good health, little or no neurological deficit, limited disease). In the first situation, treatment will be aimed exclusively at relieving symptoms; palliative therapy is based on the use of radiotherapy, analgesics, corticosteroids, anticonvulsants, ventriculoperitoneal shunts and serotonin reuptake inhibitors. In the second, the therapeutic objective is control of the neoplasm [3]. It has been shown that stereotaxic radiotherapy, used mainly in the case of nodular or symptomatic disease in the brain and/or spinal cord, and that of the brain as a whole (at the standard dose of 30 Gy in 10 fractions or 20 Gy in 5 fractions) used in the case of communicating hydrocephalus, can have beneficial effects on breast cancer symptoms. In particular, it improves the quality of life but is not associated with an increase in survival. Stereotaxic radiotherapy could limit the ability of other systemic drugs to penetrate the blood-brain barrier and in some cases cause long-term dementia [5]. In order to avoid the onset of adverse effects (myelosuppression, esophagitis, mucositis, dyspnea, diarrhea and nausea) local irradiation is usually preferred with the aim of relieving existing symptoms and avoiding the appearance of others [3]. On the other hand, intrathecal chemotherapy (with schemes based on the use of methotrexate, cytarabine and thiotepa through an Ommaya type reservoir or Rickham) appears to have an important risk-benefit ratio as it often causes neurotoxicity (aseptic, infectious or chemical meningitis, leukoencephalopathy, transverse myelopathy, myelosuppression, subdural hygromas) caused by performing these procedures, such as repeated lumbar punctures or the positioning of an intraventricular reservoir [5]. It has been shown that the latter approach is more advantageous than the former as it brings survival benefits; it is more simple to perform and safer (it allows uniform drug distribution), although not without adverse effects including infections and those related to surgery, less frequently leukoencephalopathy, increased intracranial pressure and intraventricular hemorrhage. When carrying out this procedure it is advisable to keep the volume of the cerebrospinal fluid constant to avoid complications due to a sudden change in the pressure-volume curve. Methotrexate administered intrathecally has been shown in this case to reduce the concentration of neoplastic cells with greater efficacy when associated with radiotherapy; the dosage is 10-12 mg twice a week for four weeks, adjusting the therapy according to the patient's response. An adverse effect could be myelosuppression; as it is eliminated by the kidney, this risk is increased in patients with renal insufficiency, pleural effusion, ascites and in those who take other drugs that prevent the transport of methotrexate by albumin [3]. Another intrathecal treatment option is liposomal cytarabine, 50 mg every two weeks, which has been shown to extend survival time free from neurological complications and is comparable in efficacy with methotrexate. Although its administration is of short duration, this does not exclude the possible occurrence of chemical meningitis (main adverse effect) which could be prevented by the administration of dexamethasone concomitant with chemotherapy [3]. Alternatively, although its efficacy requires further studies, it has been demonstrated that intravenous administration of thiotepa in patients with a low performance status (the most important prognostic factor) is useful [6], especially in those who have not responded to methotrexate, who have contraindications to its administration or who need concomitant radiotherapy. The dosage is $10 \mathrm{mg}$ intrathecally twice a week, adapting the duration to the patient's response; myelosuppression is the complication with which it is most often associated. The intravenous administration of these drugs, in particular methotrexate, has been shown to have advantages over the intrathecal one; for example, the absence of risk of surgical complications due to the positioning of the reservoir and the need to correct the imbalances of the cerebrospinal fluid, greater uniformity in drug distribution and disease response. The most commonly used intravenous scheme is that with high-dose of methotrexate $\left(3-8 \mathrm{~g} / \mathrm{m}^{2}\right)$ [3]. This drug often requires a hospitalization regimen to monitor plasma levels, administration of leucovorin (24 hours after chemotherapy), hydration, urinary alkalinisation (in order to prevent toxicity) and a growth factor. Some authors considered the fact that variability on drug expulsion kinetics existed among the various patients, since based on availability it is possible to determine efficacy and toxicity, both by intrathecal and intravenous administration. There are therefore two methods; one is to calculate the venous concentration of methotrexate which would give an estimate of the amount of drug eliminated (by the flow from the cerebrospinal fluid to the blood): a high and early plasma peak indicates a rapid elimination, a low and late peak shows the risk of neurotoxicity due to poor elimination. The other consists in measuring the levels of albumin in the cerebrospinal fluid, which has a negative correlation with the plasma levels of methotrexate. This can be explained considering that if the albumin concentrates in the cerebrospinal fluid not being properly expelled, consequently the same will happen with chemotherapy drugs, increasing the concentration in the intrathecal space and reducing the plasma concentration. These techniques would allow the individualization of the therapeutic dosage of the drug, favoring the correct availability and avoiding the toxic effects [7]. In the case of overdose, the treatment consists in the administration of glucarpidase intrathecally associated with ventricolo-lumbar perfusion in order to reduce its concentration. If repeated administrations of methotrexate are necessary, some authors say it may be useful to identify patients with an increased risk of toxicity by measuring the activity of methylenetetrahydrofolatereductase (MTHFR) by genotype, the reduction of which could favor the appearance of side effects. In addition, intravenously administered capecitabine would also be beneficial, increasing the therapeutic response and stabilizing the disease [3].

In some studies, intrathecal trastuzumab alone or in combination with other systemic drugs in clinical, cytological, radiographic situations showed improvement without serious adverse effects; this was highlighted in patients with HER-2 positivity [8]. The use of some drugs is hindered in the passage from the blood-brain barrier, not only due to the presence of astrocytic junctions that have a protective role on the barrier but also of the p-glycoprotein whose function is to expel from the brain all toxic substances; therefore, the same barrier between blood and cerebrospinal fluid could represent an alternative for pharmacological transport, thanks to the presence of the choroid plexus whose main functions are brain homeostasis and metabolism. Some strategies are being studied which, on the one hand, would facilitate the transport of chemotherapeutic agents to the cerebrospinal fluid by exploiting the properties of the choroid plexus (for example, the presence of the prolactin receptor which would favor the transport 
of anticancer agents between blood and cerebrospinal fluid, $\mathrm{Na}+1$ ascorbate co-transporter, viruses that infect the epithelium and leukocyte trafficking through the plexus). On the other hand, these strategies could prevent the access of tumor cells by blocking the markers present on its surface. The presence of a C3a receptor produced by the same choroid plexus has been demonstrated in patients with leptomeningeal metastases secondary to mammary neoplasia whose block would prevent the entry of tumor growth factors into the cerebrospinal fluid and therefore their metastatic dissemination [2].

Finally, the efficacy of the combination of therapeutic regimens, such as intrathecal topotecan combined with intravenous eribulin, has been demonstrated, showing radiological stability of the disease and slight clinical improvement without serious systemic toxicities [9]. Hormone therapy with tamoxifen, letrozole and examestane that, in cases of breast cancer with positive estrogen and progestogen receptors, slow growth and small size would favor an increase in survival without causing serious side effects [10] has also been considered. VEGF (the growth factor of the vascular endothelium) has been identified as an element of poor prognosis which would favor the dissemination of disease. The role of bevacizumab, a human monoclonal antibody that acts against it, has thus been investigated, bringing not only changes in the blood brain barrier but also favoring the reduction of VEGF concentration in the cerebrospinal fluid. This drug could generate beneficial effects on tumor vascularity, facilitating the penetration of other chemotherapeutic agents into the neoplasm for which the combination of bevacizumab and chemotherapy after a few days has been shown to lengthen the disease-free progression (decrease in leptomeningeal dissemination with calcifications, transient disappearance of tumor cells from the cerebrospinal fluid and improvement of neurological deficits) with few secondary effects, the most frequent comorbidity being cerebral hemorrhage [11].

Finally, as regards the prognosis, additional factors that influence a negative response to treatment are: late diagnosis, the presence of irreversible neurological deficits and an advanced disease stage as well as the molecular characterization of triple negative neoplasia. On the contrary, elements that favor the prognosis are: young age and good performance status, long duration of pre-treatment symptoms and good control of disseminated disease. In general, the survival of these patients is unfortunately limited to a few weeks, even though they belong to the low-risk class [3].

\section{Conclusion}

Although there are various therapeutic strategies in the context of meningeal carcinomatosis, there is no standardized scheme. As evidence of efficacy is poor, it would be advisable to individualize the treatment according to the patient's needs, applying a multidisciplinary approach and performing more prospective studies. The use of target therapies should also be considered.

\section{References}

1. Pavlidis N (2004) The diagnostic and therapeutic management of leptomeningeal carcinomatosis. Ann Oncol 15: iv285-iv291. [Crossref]

2. Franzoi M, Hortobagyi G (2019) Leptomeningeal carcinomatosis in patients with breast cancer. Crit Rev Oncol Hematol 135: 85-94. [Crossref]

3. https://www.uptodate.com/contents/treatment-of-leptomeningeal-metastasescarcinomatous-meningitis

4. Kapke JT, Schneidewend RJ, Jawa ZA, Huang CC, Connelly JM, et al. (2019) Highdose intravenous methotrexate in the management of breast cancer with leptomeningeal disease: case series and review of the literature. Hematol Oncol Stem Cell Ther 12: 189-193. [Crossref]

5. Mrugala MM, Kim B, Sharma A, Johnson N, Graham C, et al. (2019) Phase II study of systemic high-dose methotrexate and intrathecal liposomal cytarabine for treatment of leptomeningeal carcinomatosis from breast cancer. Clin Breast Cancer 19: 311-316. [Crossref]

6. Chahal J, Stopeck A, Clarke K, Livingston RB, Chalasani P (2015) Intravenous thiotepa for treatment of breast cancer-related leptomeningeal carcinomatosis: case series. Neurol Sci 36: 1691-1693. [Crossref]

7. Huber M, Nolting M, Bauer S, Leistner S, Schmitz U, et al. (2005) Approaching an individual methotrexate regimen in leptomeningeal carcinomatosis. J Neurol Sci 236 : 37-41. [Crossref]

8. Assi HI, Mahmoud T, Saadeh FS, El Darsa H (2018) Management of leptomeningeal metastasis in breast cancer. Clin Neurol Neurosurg 172: 151-159. [Crossref]

9. Salgia S, Fleming GF, Lukas RV (2014) Leptomeningeal carcinomatosis from breast cancer treated with intrathecal topotecan with concomitant intravenous eribulin. $J$ Clin Neurosci 21: 1250-1251. [Crossref]

10. Takanashi T, Hikino H, Makino Y, Murata Y (2019) Durable clinical benefit of letrozole in leptomeningeal metastasis of breast cancer. Int Cancer Conf J 8: 146-148. [Crossref]

11. Chen IC, Lin CH, Jan IS (2016) Bevacizumab might potentiate the chemotherapeutic effect in breast cancer patients with leptomeningeal carcinomatosis. J Formos Med Assoc 115: 243-248. [Crossref]

Copyright: (C2020 Marasciulo F. This is an open-access article distributed under the terms of the Creative Commons Attribution License, which permits unrestricted use, distribution, and reproduction in any medium, provided the original author and source are credited. 